Techniques \& Culture

$61 \mid 2013$

Vivre le sable !

\title{
Marcher au Japon
}

The Walking to Japan

\section{Augustin Berque}

\section{OpenEdition}

Journals

Édition électronique

URL : https://journals.openedition.org/tc/7327

DOI : $10.4000 /$ tc. 7327

ISSN : 1952-420X

\section{Éditeur}

Éditions de l'EHESS

\section{Édition imprimée}

Date de publication : 15 décembre 2013

Pagination : 254-269

ISBN : 978-2-7351-1654-6

ISSN : 0248-6016

\section{Référence électronique}

Augustin Berque, « Marcher au Japon », Techniques \& Culture [En ligne], 61 | 2013, mis en ligne le 15 décembre 2016, consulté le 29 septembre 2022. URL : http://journals.openedition.org/tc/7327 ; DOI : https://doi.org/10.4000/tc.7327 


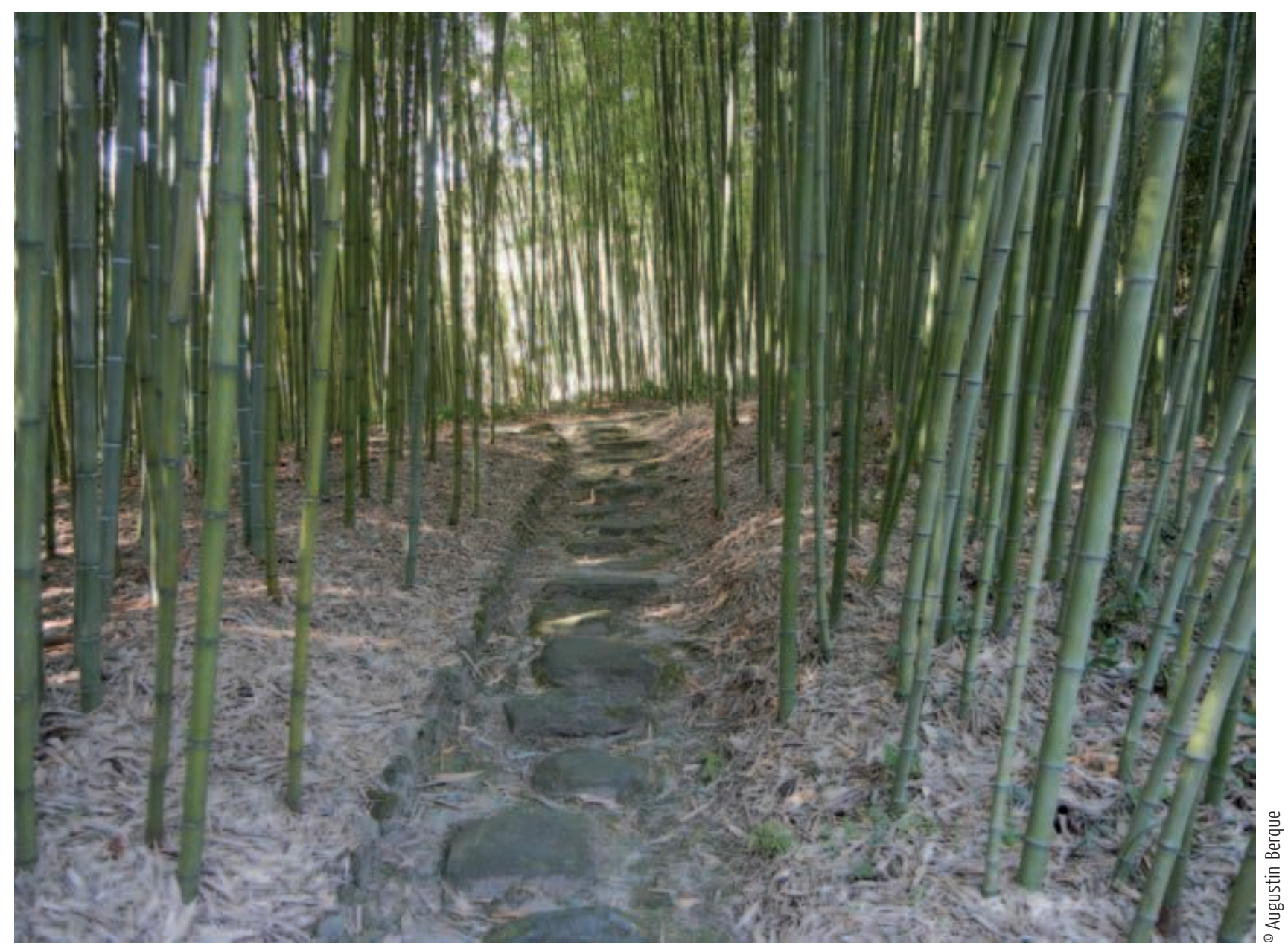


Augustin Berque

EHESS

berque@ehess.fr
Vivre le sable! Corps, matière et sociétés

Techniques \& Culture 61, 2013/2: 254-269

\section{MARCHER AU JAPON}

Le texte qui suit est une version adaptée pour la revue Techniques \& Culture de la Conférence donnée par Augustin Berque le 10 octobre 2013 à l'École spéciale d'architecture de Paris dans le cadre du Cycle de conférences de l'automne 2013: La Marche.

\section{Le chemin du Bois de bambous}

L'image ci-contre est une photographie que j’ai prise il y a quelques années au parc du Bois de bambous de Kyôto (Kyôto-shi Rakusai Chikurin kôen 京都市洛西竹林公園). C'est un parc où est rassemblée une grande variété d'espèces de bambous, de tailles et de formes parfois surprenantes. Le diamètre des plus gros bambous atteint une quinzaine de centimètres, et ils peuvent dépasser les vingt mètres de hauteur. C'est le cas de l'espèce qu'on appelle au Japon madake 真竹 (Phyllostachys ${ }^{1}$ bambusoides), le « vrai bambou ». Ceux que vous voyez sur l'image sont d'une autre espèce, plus petite, le bambou de Budai (Hoteichiku 布袋竹, Phyllostachys aurea). Le bambou, notamment le madake, est une plante naturelle au Japon, mais que l'on cultive aussi parce qu'elle se prête à de très nombreux usages, y compris alimentaires (les « pousses de bambou», takenoko 竹の 子). Au petit Musée du bambou qui est adjoint au parc, on apprend même que dans les dernières années de la guerre du Pacifique, par manque de métal, on avait mis au point du béton armé par des tiges de bambou! 
Pour en venir au thème de la marche, on pourrait mentionner que ce qu'on appelle en français des échasses se dit en japonais « cheval de bambou », take uma 竹馬 ; mais ce n'est pas pour cette raison que j'ai choisi cette image, c'est à cause du chemin. C'est un chemin qui, comme vous le voyez, n'est pas ordinaire. Il a été travaillé pour un effet esthétique, avec ses pierres du genre qu'on appelle, dans l'art des jardins, «pierres traversières», watari ishi 渡り石 (ce qu'on traduit souvent par « pas japonais »). On les utilise en particulier dans le jardin de thé, roji 露地. Leur nom signifie littéralement « pierres » (ishi 石) pour « traverser» (wataru 渡る). Pour traverser quoi ? Le jardin, apparemment, jusqu'à la cabane à thé (chashitsu 茶室). Ces pierres, effectivement, ont cette fonction, mais ce n'est pas la seule. D'autres également sont en jeu. C'est ce que rappelle un adage qui met en scène deux grands maîtres de l'art du thé, comme de l'art des jardins qui en est indissociable, Sen no Rikyû ${ }^{2}$ (1522-1591) et son disciple Furuta Oribe (1543-1615). La tradition rapporte qu'à propos de ces pierres traversières, Rikyû aurait préconisé « six parts de traversée, quatre parts de décor» (watari roku bu, keiki yon bu 渡り六分、景気 四分), mais Oribe «six parts de décor, quatre parts de traversée » (keiki roku bu, watari yon bu景気六分、渡り四分) (Berque [1986] 1997). Outre la marche, il y a là évidemment aussi du décor, ou de la décoration ; mais ce n'est pas tout encore. Le mot qui veut dire «traverser», wataru渡る, s'écrit avec un sinogramme qui comporte à sa gauche la clef de l'eau, sous forme de trois gouttes. C'est qu'effectivement, il s'agit ici, à l'origine, de traverser un cours d'eau, plus exactement un torrent montagnard, en sautant de pierre en pierre. C'est cela qu'évoquent les pierres traversières, et c'est pour cela que, dans le jardin de thé, on les dispose de manière irrégulière.

Traverser un torrent, mais pourquoi donc ? C'est que l'espace-temps de la cérémonie du thé, le chanoyu 茶の湯, relève d'un double corpus mythologique. L'un provient du bouddhisme. Nous en avons l'indice dans un propos de Rikyû, rapporté par ses disciples dans le Nambôroku 南方録, cette Bible de l'art du thé (qui semble avoir été composée plus tard, en réalité) :

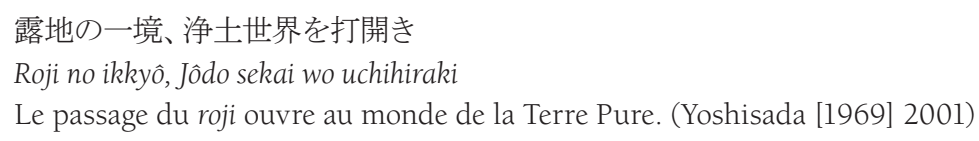

ce qui signifie que le jardin, qu'il faut traverser pour accéder à la cabane à thé, symbolise le passage de la frontière ${ }^{3}$ entre le domaine mondain et la Terre Pure ${ }^{4}$, c'est-à-dire le paradis bouddhique, Sukhāvati. Corrélativement, la cabane à thé, aboutissement de ce passage, symbolise la Terre Pure, au-delà des poussières mondaines (l'extérieur du jardin). Sur l'image de l'affiche, nous ne sommes pas dans un jardin de thé, mais on pourrait dire que la Terre Pure, c'est la clairière - la Lichtung, comme dirait Heidegger - que l'on voit au fond : une ouverture de monde.

Ce n'est pas tout. Le passage dont parle Rikyû est une métaphore concrète, non point seulement verbale. Cette métaphore, d'un côté, à l'échelle intra-urbaine voire domestique, s'incarne dans le roji ; mais d'un autre côté, à l'échelle territoriale, elle implique la réalité des espaces de l'Asie orientale, dans leur lien entre étendue physique et religion bouddhique. Concrètement donc, la Terre Pure est à l'ouest; c'est le « paradis occidental $»^{5}$. Le chemin ardu qu'il faut parcourir pour y accéder, ce sont les déserts et les montagnes géantes que Xuan Zang, au vile siècle, dut affronter pour aller chercher les Écritures en Inde (Grousset 1929). En passant par un roman d'aventures du Xvi e siècle, la Pérégrination vers l'Ouest (Xiyouji 西遊記) (Lévy 1991), c'est l'origine de la célèbre 


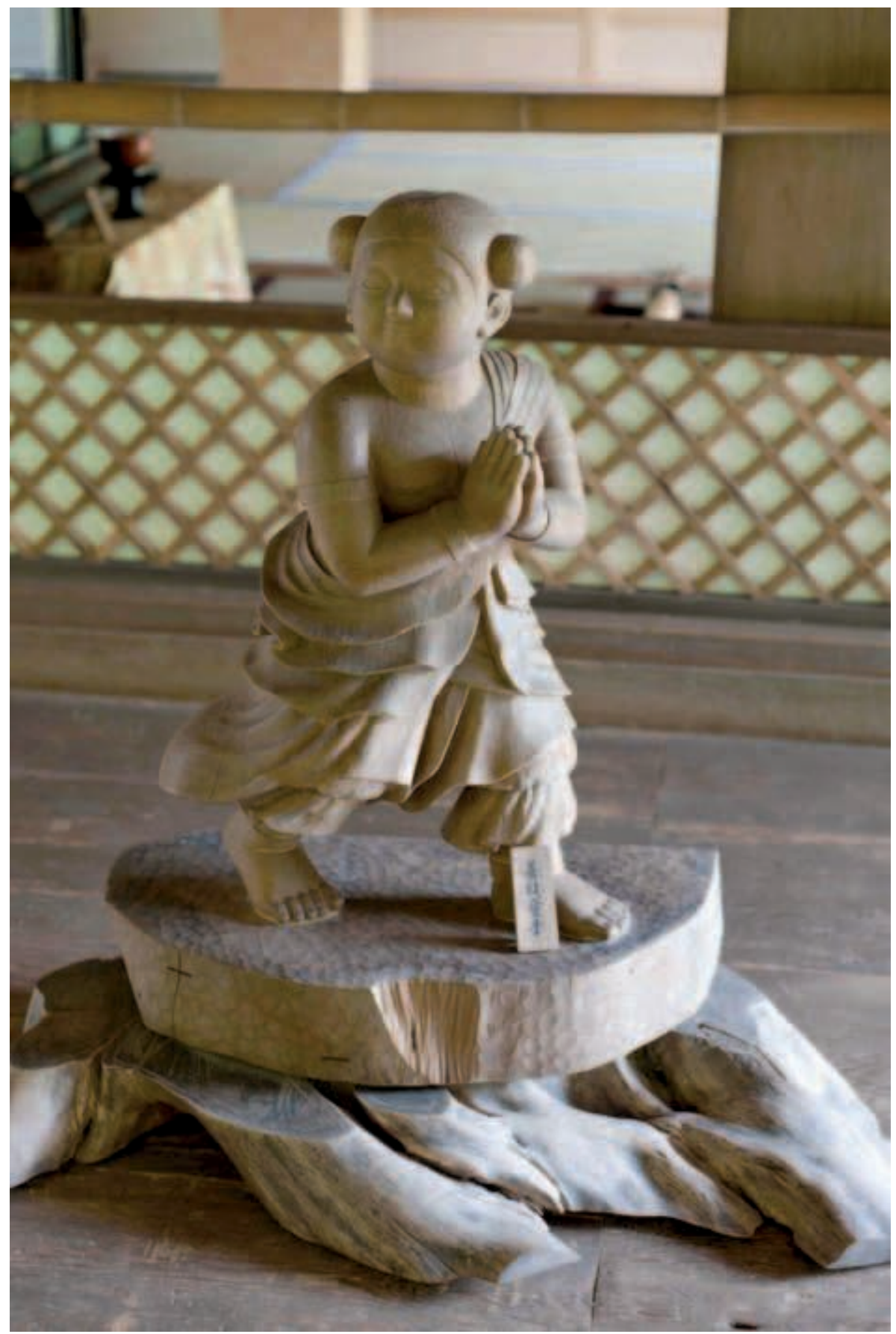

Shancai (Sudhana), disciple du Bouddha, fameux pour avoir cherché dès son jeune âge l'illumination sous la direction de 53 maitres, ce dont les 53 étapes du Tôkaidô sont une métaphore. 
manga de Toriyama Akira, Dragon ball. Ces terres du Far West de la Chine, ce sont aussi les montagnes du dispositif cosmique « haut à l'ouest, bas à l'est » (xi gao dong di 西高 東低) du monde chinois, qui culmine au Kunlun... Certes, le Kunlun ne doit rien au bouddhisme ; mais concrètement, il fait cause commune avec la montagne cosmique imaginée en Inde, le Sumeru ${ }^{6}$; laquelle, véhiculée par le bouddhisme, a été reproduite à profusion dans les jardins japonais.

Dans la cosmologie du jardin de thé, nous avons donc l'homologie structurale suivante, qui va nous conduire au second corpus mythologique, celui de l'érémitisme mandarinal :

Traversée du jardin $=$ marche vers la Terre Pure $=$ remontée vers l'ouest $=$ anachorèse.

Ce mot savant, " anachorèse », plus simplement " retraite », nous vient du grec

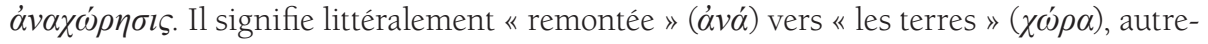
ment dit quitter le monde, dont la ville est le nombril. Ce thème a connu en Chine une vogue extraordinaire sous les Six Dynasties ( $\mathrm{III}^{\mathrm{e}}-\mathrm{VI}^{\mathrm{e}}$ siècle), époque troublée où les mandarins avaient souvent à se réfugier dans leurs terres, pour manifester leur désaccord avec le monarque ou pour sauver leur peau. Ces lettrés en ont bâti un mythe analogue à la Pastorale en Occident, celui de l'ermitage paysager (yindun 隠遁). « Paysager », car ce sont eux en effet qui ont inventé la notion de paysage (shanshui 山水), mot dont le sens originel, venu de l'hydraulique, était jusque-là celui de « les eaux de la montagne », c'est-à-dire « torrent montagnard ». Ces torrents, c'est ce qu'il fallait traverser, en sautant d'une pierre à l'autre, pour atteindre l'ermitage où un mandarin anachorète avait fait retraite. D'où plus tard, dans les jardins japonais, l'autre nom des pierres traversières : tobi ishi 飛び石, «pierres à sauter».

À travers la poésie chinoise classique, dite en japonais tôshi 唐詩 (《poésie Tang ») ou plus généralement kanshi 漢詩 ( poésie Han »), la mythologie de l'ermitage paysager a exercé une profonde influence sur l'esthétique japonaise, en particulier sur l'art des jardins et sur l'architecture (Berque 2010). Si ce que nous appelons « pavillon » (de thé) veut dire en réalité « cabane » (à thé), an ou iori (庵), c'est parce que son archétype est la cabane en pleine nature dont les anachorètes lettrés des Six Dynasties ont construit le mythe, sinon la réalité. Et si aujourd'hui, par dizaines de millions, dans les maisons individuelles des banlieues japonaises, le tokonoma 床の間 (renfoncement où l'on accroche une décoration, calligraphie, fleur ou paysage) est jouxté d'une colonne (le tokobashira 床柱) qui affecte un aspect naturel - au point même de garder parfois son écorce, dans l'architecture du thé-, c'est parce que cette colonne, et à travers elle toute la maison, fait allusion à cette cabane faite de branchages qu'était censé être l'ermitage paysager des Six Dynasties, loin des poussières mondaines. C'est pourquoi je traduis tokobashira par « colonne érémitique ».

Revenons à l'image du bois de bambous. Les mandarins anachorètes des Six Dynasties se sont eux-mêmes appelés yinzhe 隠者, « ceux qui se cachent ». Beaucoup furent poètes, musiciens, écrivains ou philosophes. Bien que fuyant le monde, ces mandarins excellaient dans certaines activités proprement mondaines, telles notamment les « pures causeries », qingtan 清談. Entré dans l'usage à l'époque Han, ce terme a désigné d'abord des propos élégants, tenus entre personnages distingués à l'écart du monde vulgaire. Sous les Six Dynasties, il va prendre plus particulièrement le sens de : «propos philosophiques » dans la mouvance taoïste. Les plus célèbres pratiquants de cet art de converser furent les Sept Sages du Bois de bambous ${ }^{7}$, un cercle d'anachorètes qui vécurent au III $^{\mathrm{e}}$ siècle de notre ère, vers la fin du royaume Wei et la réunification momentanée de l'empire 
par les Jin ${ }^{8}$. La tradition en a retenu que " profondément liés entre eux, ils fuyaient le monde et s'amusaient dans les bambous ${ }^{9}$ (Qingyuan \& al. 1998). Le bois de bambous que vous voyez sur l'image m'a bien sûr fait penser à eux ; mais plus directement, il m'a fait penser à un personnage plus ancien, Jiang $\mathrm{Xu}$, qui s'était retiré dans un bois de bambous pour protester contre l'usurpation de Wang Mang (9-23), sous les Han. Il y avait aménagé trois sentiers, où il se promenait avec deux amis, comme lui retraités, pour protester contre l'usurpateur. Depuis, dans toute l'Asie orientale, l'expression « trois sentiers » (san jing 三徑) est devenue symbole de l'amitié fidèle. C'est cela d'abord que j’ai tenu à photographier, pour cette affiche. Vous savez maintenant quoi faire, si vous aimez la marche et voulez garder vos amis..

\section{Marcher à l'amble ou en crabe ?}

Ce chemin du Bois de bambous nous entraîne loin du Japon d'aujourd'hui, me direzvous. En apparence seulement! Primo, comme le montre la tradition du tokobashira, cette histoire est toujours vivante, même si la plupart des Japonais en sont inconscients. Ils l'ont en effet complètement japonisée, et hormis quelques spécialistes, ils pensent qu'elle a son origine dans les campagnes japonaises. La cabane à thé aurait imité les masures paysannes du Japon lui-même. En réalité, ce fut l'inverse : imaginée d'abord en Chine par une élite mandarinale, cette cabane, sous forme de poésie, est entrée au Japon par la Cour impériale à l'époque de Heian (IXe-XII siècle), et c'est de là que, petit à petit, elle est descendue dans la bonne société nippone. L’apparente rusticité de son architecture n'a rien de paysan, c'est en quelque sorte l'analogue du Petit Trianon de Marie-Antoinette.

Secundo, de tous les aménagements humains, les chemins sont parmi ceux qui résistent le plus aux changements de l'histoire. Si beaucoup de rues de Tokyo sont sinueuses, c'est parce que ce sont d'anciens chemins ruraux, comme l'a bien montré l'architecte Jinnai Hidenobu, dans un livre sur la spatialité de Tokyo qui a été traduit en anglais et que vous pouvez donc lire (Jinnai 1995). Pour sentir cette spatialité, la méthode de Jinnai consiste avant tout à marcher dans la ville. Il l'a utilisée non seulement au Japon, mais aussi dans des villes étrangères, notamment en Chine et en Italie. Il appliquait en somme ce précepte que m'a transmis le géographe Jean Delvert, qui dirigea ma thèse : «Un géographe, ça pense avec ses pieds ». J'ai mis longtemps à digérer ce précepte, parce qu'il me laissait craindre que les géographes, par rapport à des anthropologues ou à des sociologues, par exemple, ne fussent bêtes comme leurs pieds. Bien entendu, l'inverse est plus vraisemblable : les géographes, selon ce principe, auraient une intelligence du pied digne d'un cerveau humain. Et en fait, nous en sommes tous là : nous pensons de tout notre corps, de la tête aux pieds, et des pieds à la tête. C'est pourquoi la marche est favorable à la méditation. Un linguiste et un cogniticien américains en ont tiré, voici une douzaine d'années, un livre qui s'intitule tout simplement : Philosophie dans la chair (Lakoff \& Johnson 1999).

Tertio, hier comme aujourd'hui, « marcher » se dit en japonais aruku 歩<. Le mot est ancien. On le trouve déjà dans le Manyôshû 万葉集, le plus ancien monument de la littérature japonaise. Mais si ce mot est resté à peu près tel quel depuis les premiers écrits en langue japonaise, ce qu'il recouvre a-t-il toujours été ce que nous connaissons 


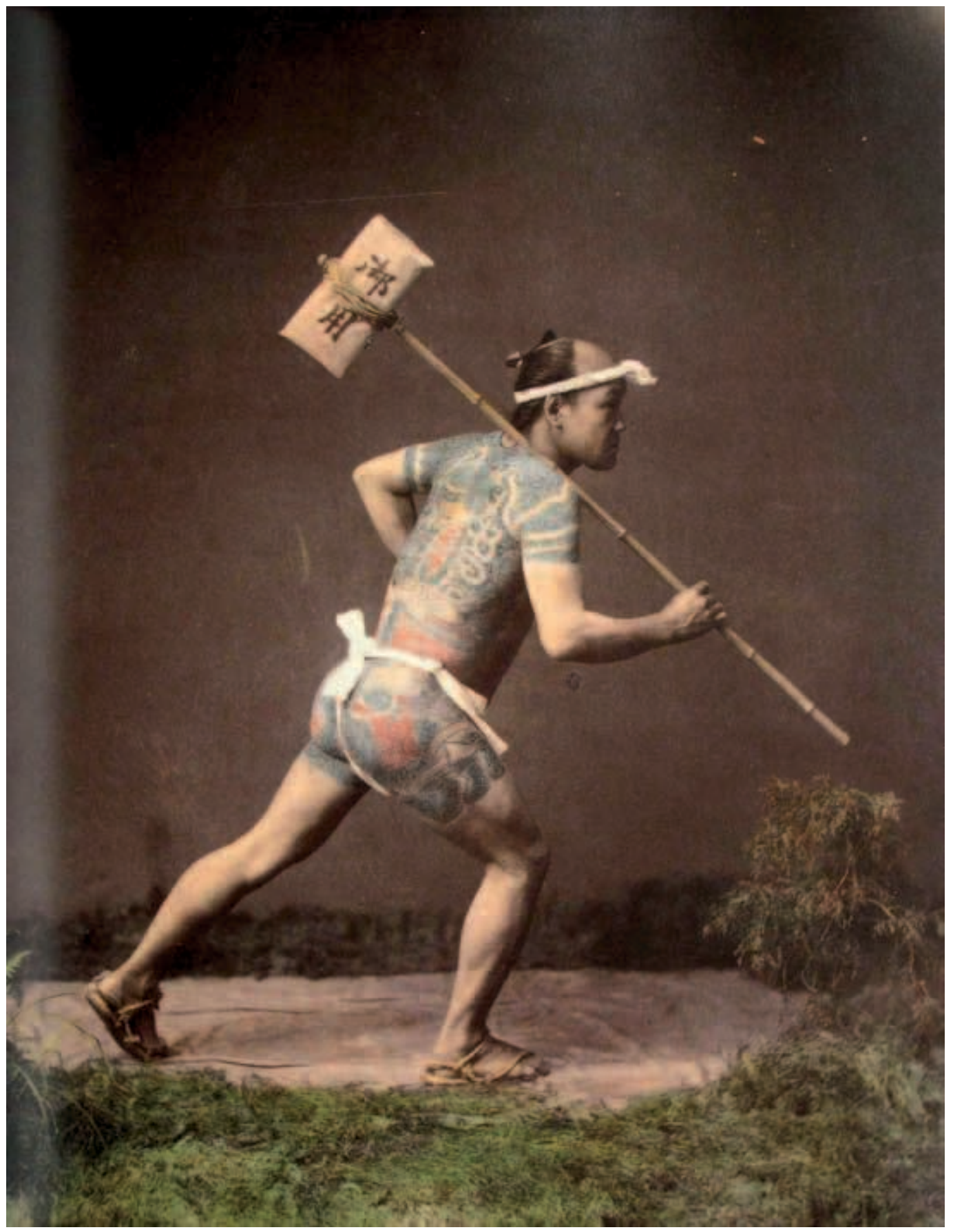

Coureur tatoué Les caractères sur la missive quill porte, goyô 御用, signifient qu'il est en mission officielle. 
aujourd'hui ? Peut-être pas. Aujourd'hui, les Japonais marchent à peu près comme nous, à de menues différences près, dont je dirai un mot tout à l'heure. Il se pourrait cependant qu'ils aient marché autrefois d'une autre manière, comme le laissent soupçonner deux expressions : Nanba aruki ナンバ歩き et Nanbabashiri ナンバ走り, ce qui veut dire respectivement «marche à la Namba » et « course à la Namba ». Nanba est ici le plus souvent écrit dans le syllabaire katakana, non pas en sinogrammes; mais en sinogrammes, cela s'écrit 難波, ce qui est un toponyme correspondant à Ôsaka, dont c'est aujourd'hui le nom de l'un des arrondissements centraux. Ses deux sinogrammes sont ici des ateji 当 て字, c'est-à-dire des caractères sans rapport sémantique avec la prononciation du mot qu'ils représentent. Littéralement, ils signifient « vague difficile»; mais il s'agit plus vraisemblablement d'une déformation de Nagiwa 波際, « au bord des vagues » autrement dit « littoral », ce qui correspond effectivement au lieu en question.

La « course à la Namba », Nanbabashiri serait la manière dont couraient les « pieds volants », hikyaku 飛脚, messagers qui faisaient à pied la liaison entre la capitale administrative, Édo (aujourd'hui Tokyo) et la région du Kamigata (Kyôto et Ôsaka) par l'itinéraire du Tôkaidô (東海道, « voie des mers de l'est »). D'Édo à Kyôto, en 53 étapes, la distance était de $495 \mathrm{~km}$. D’Édo à Ôsaka, en 57 étapes, elle était de $546 \mathrm{~km}$. Au tarif du courrier ordinaire, les coureurs mettaient un mois pour couvrir cette distance, soit entre $15 \mathrm{et}$ $20 \mathrm{~km}$ par jour. Le tarif express était appelé « dans les quatre jours», yokkagiri 四日限, ce qui impliquait des étapes quotidiennes de plus de $100 \mathrm{~km}$. Pour ménager leur corps, ces coureurs auraient adopté une démarche spéciale, le Nanbabashiri, qui aurait été ce que l'on appelle en français l'amble (ce mot vient du latin ambulare, marcher). Chez les animaux, c'est la façon de marcher naturelle aux chameaux, aux girafes, aux ours etc., qui avancent en même temps les deux pattes du même côté. Le cheval aussi peut aller l'amble, mais il faut le lui apprendre. Chez l'homme, cela consiste à avancer en même temps pied droit et main droite, puis pied gauche et main gauche. Cette démarche aurait eu l'avantage d'augmenter l'enjambée. Cependant, avec la disparition des « pieds volants ", la tradition s'est perdue sous Meiji, et il ne subsiste aucun texte ou dessin qui la décrive expressément.

Il existe une interrogation identique quant à la « marche à la Namba », Nanba aruki. Là aussi, l'avantage aurait été d'allonger l'enjambée. On dit encore que cela aurait facilité le travail du paysan, notamment le désherbage, tâche la plus lourde dans un climat comme celui du Japon. Mais là aussi, la tradition s'est perdue, et l'on manque de documents précis. Les estampes de l'époque d'Édo, puis les photographies et surtout les films, ne permettent pas de juger si l'amble, autrefois, a bien été la démarche normale des Japonais. L'on y voit plutôt des gens qui marchent comme aujourd'hui.

Reste qu'aujourd'hui encore, j'ai l'impression qu'il y a bien une certaine façon japonaise de marcher, que je n'ai pas remarquée ailleurs. C'est difficile à définir, mais si l'on a la question en tête, cela pourrait vaguement évoquer l'amble, dans la manière de déplacer le bassin ; mais je n'ai pas de documentation sérieuse sur cette question.

À côté de cela, il y a les déformations qu'entraînent l'usage du tatami et la manière correcte de s'y agenouiller, le seiza 正座, dans lequel la cheville est à la fois allongée à $180^{\circ}$ et tordue vers l'intérieur à $90^{\circ}$; ce qui fait qu'une fois debout, les Japonais et surtout les Japonaises ont tendance à marcher les pieds en dedans. L'usage du tatami a aussi engendré des générations de «jambes de crabe », ganimata 蟹股, où la déformation du tibia donne à la jambe l'allure d'un point d'interrogation à l'envers. Cela ne manque pas de retentir sur la démarche. Depuis que le kimono a laissé place à la jupe, la jambe de 
crabe a été un souci permanent pour les Japonaises (cela se voit moins chez les hommes, grâce au pantalon), jalouses des jolies jambes chinoises ou coréennes. Aussi les jeunes générations évitent-elles autant que possible le seiza, ce qui leur est du reste facilité par la transformation du mobilier. Aujourd'hui, même chez soi, l'on est beaucoup plus souvent assis sur une chaise qu'agenouillé sur un tatami. Cependant le problème existe encore, et alimente les instituts de beauté ou de huittonesu フイットネス (fitness) où l'on vous apprend à corriger votre 《 démarche de crabe » (ganimata aruki 蟹股歩き) - ce qui ne veut pas dire qu'on se déplace latéralement, comme les crabes, mais avec les pieds exagérément en dedans, surtout le droit, qui dans le seiza féminin se trouve au-dessus du pied gauche, et donc encore plus tordu (pour les hommes, c'est l'inverse : le gauche au-dessus).

\section{Une culture du cheminement ?}

Que la marche ou la course à la Namba aient été plus ou moins généralisées dans le Japon d'autrefois reste conjectural, mais ce qui est certain, c'est que les grands itinéraires d’avant Meiji, les kaidô 街道, étaient des chemins pédestres. On pouvait également y aller à cheval, mais sans voiture. Le Japon était un cas de pays peu commun qui, bien qu'utilisant la roue, s'en est peu servi pour les transports de personnes. Cela a sans doute un rapport avec le caractère montagneux du pays. Avant Meiji, la règle était que le peuple aille à pied, les élites en palanquin ou à cheval. Le transport des pondéreux se faisait par voie d'eau. La seule exception notable est celle des chars à boufs (gissha 牛車) que l'on utilisait depuis l'Antiquité dans la région de Kyôto, la capitale impériale, tant comme carrosses pour la noblesse de Cour que pour transporter les marchandises entre Heian (l'ancien nom de Kyôto) et le port d'Ôtsu, sur le lac Biwa. Mais curieusement, cet usage ne s'est pas répandu dans le reste du pays.

C'est toute la territorialité japonaise qui a été dictée par cette prépondérance de la marche, à tel point que le paysagiste Yoshimura Motoo a pu parler de hokôsha no bunka 歩行者の文化, « culture du piéton » (Yoshimura 1976). Le meilleur exemple qu’on puisse en donner, c'est l'itinéraire du Tôkaidô, avec les 53 étapes (gojûsan tsugi 五十三次) immortalisées par les estampes de Hiroshige. Je reproduis à la page suivante la liste des dix premières, à partir du pont Nihonbashi à Édo, que l'on voit sur l'estampe de Hiroshige reproduite également (source : 350ml.net/labo/ tokaido.html). Comme le parvis de Notre-Dame en France, Nihonbashi (le Pont du Japon, ou du Soleil Levant) est le point de départ de tous les itinéraires du Japon, celui à partir duquel on compte les distances. Sous Meiji, on y construisit un pont monumental, qui évoque un peu le pont Alexandre III à Paris. Si l'on met à part le palais impérial, non loin de là, c'est ou plutôt c'était le repère essentiel, le cour du pays. C'était, parce que l'économicisme triomphant des années de Haute Croissance a fait passer une autoroute par-dessus. Triste symbole...

On voit sur la liste ci-après que les étapes du Tôkaidô n'étaient jamais à plus d'une quinzaine de kilomètres l'une de l'autre, soit 4 ou 5 lieues japonaises ( $r i$ 里). C'est une distance adaptée à la marche. La suite des étapes était exprimée par le mot tsugi次 (suite, suivant), mais les étapes elles-mêmes s'appelaient shukuba 宿場, « lieu d'hébergement ». Shuku, dont le sinogramme 宿 se lit également yado (logis, auberge), se retrouve dans de nombreux toponymes au Japon; par exemple, à Tokyo, Shinjuku 新宿, ce qui veut dire « la Nouvelle-Étape », que l'on aménagea au xviII siècle sur l'itinéraire du Kôshû (Kôshû 


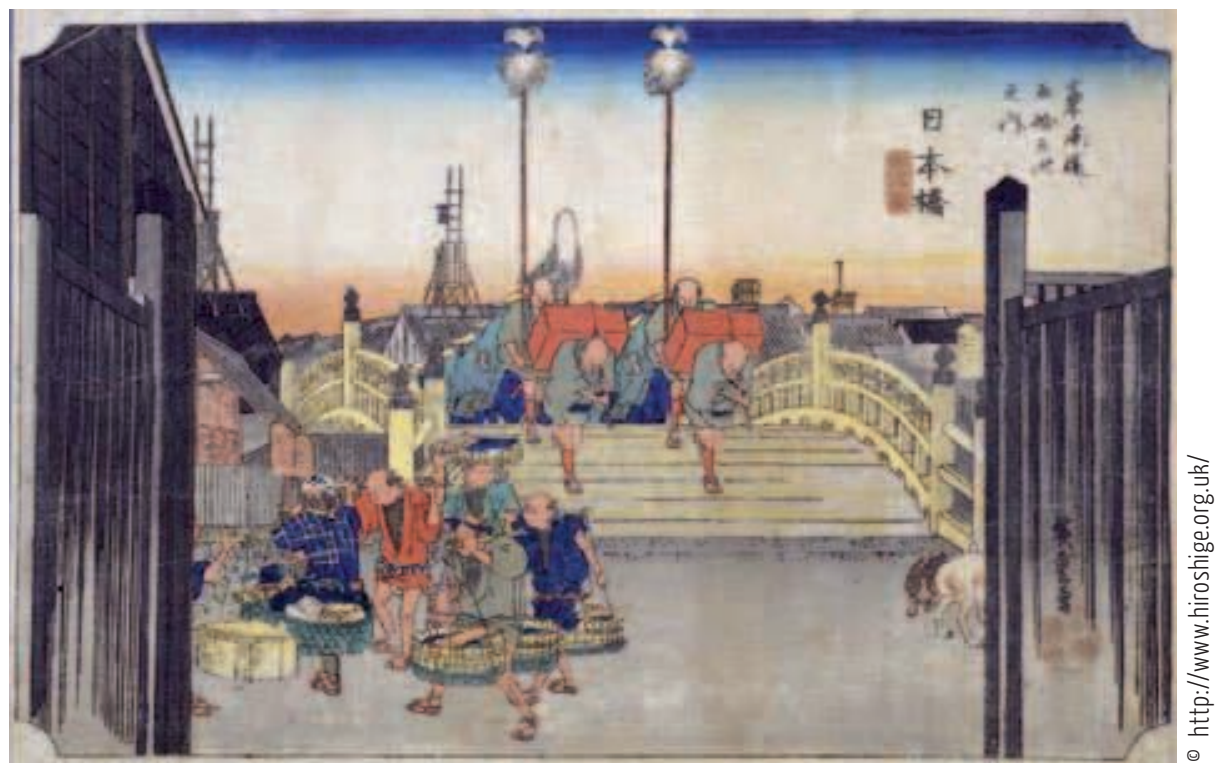

kaidô 甲州街道). Shinjuku est depuis longtemps l'un des quartiers les plus animés de Tokyo, et il a accueilli en 1991 le nouveau siège de la Préfecture, œuvre fameuse de Tange Kenzô. La plupart des étapes des grands itinéraires ont été en effet des noyaux d'urbanisation, parce que chacune attirait toutes sortes d'activités liées au voyage.

C'est là un aspect essentiel de la culture japonaise, qu'on pourrait à plusieurs égards qualifier de « culture du chemin », michi no bunka 道の文化, voire, plus précisément, de michiyuki no bunka 道行の文化, « culture du cheminement». Cette notion de cheminement (michiyuki) a été, entre autres, à l'origine d'un genre littéraire auquel la grande japonologue Jacqueline Pigeot a consacré sa thèse, Michiyuki-bun. Poétique de l'itinéraire dans la littérature du Japon ancien. Le mot michiyuki-bun 道行文, qui signifie « littérature (bun) d'itinéraire (michiyuki)», a été créé par la philologie moderne pour désigner un type de littérature qui se développa au xiII ${ }^{e}$ siècle et connut une grande faveur à l'époque Muromachi (XIVe-XVI ${ }^{e}$ siècles). Il s'agit de la relation de voyages fictifs dans des lieux connus, dont le style se caractérise par une prose rythmée (surtout des heptamètres et des pentamètres alternés, comme dans le haïku), évoquant la nostalgie du voyageur, riches en toponymes et en citations de poèmes anciens, et mettant systématiquement en œuvre des tropes appelés engo 縁語 et kakekotoba 掛詞.

Le procédé de l'engo, littéralement « mot (go) qui a un rapport (en)», consiste à insérer un mot relevant du même champ sémantique qu'un autre mot qui le précède, en lui donnant une valeur métaphorique. C'est ainsi que, dans l'exemple tiré du Kokinshû que Pigeot analyse p. 106, Akigiri no / tomo ni tachi idete 秋霧のともに立出でて..., tachi est relatif à akigiri (brouillard d'automne). Il peut avoir le double sens de « s'élever » (au sens de « le brouillard qui s'élève ») ou de " partir» (dans le sens de « l'être aimé qui s'en va »). Il y a donc en français le choix entre deux traductions possibles mais mutuellement exclusives : « Avec le brouillard d'automne qui s'élève... » et « Avec le brouillard d'automne, si vous partez... » alors que dans le texte japonais, les deux interprétations sont simultanément présentes et compossibles. 


\section{Les dix premières des 53 étapes du Tôkaidô}

\begin{tabular}{|c|c|c|c|}
\hline 宿場名 Nom de l'étape & 現在の住所 Adresse actuelle & $\begin{array}{l}\text { 日本橋からの距離 } \\
\text { Distance à partir de } \\
\text { Nihonbashi }\end{array}$ & $\begin{array}{l}\text { 次の宿場までの距離 } \\
\text { Distance jusqu'à l'étape } \\
\text { suivante }\end{array}$ \\
\hline $\begin{array}{l}\text { 江戸 (日本橋) Édo } \\
\text { (Nihonbashi) }\end{array}$ & 東京都中央区 & - & 2 里 $r i$ (lieues) $(7,9 \mathrm{~km})$ \\
\hline 品川 Shinagawa & 東京都品川区 & 2 里 $(7,9 \mathrm{~km})$ & 2.5 里 $(9,8 \mathrm{~km})$ \\
\hline 川崎 Kawasaki & 神奈川県川崎市川崎区 & 4 里 18 町(17,7 km) & 2.5 里 $(9,8 \mathrm{~km})$ \\
\hline 神奈川 Kanagawa & 神奈川県横浜市神奈川区 & 7 里 $(27,5 \mathrm{~km})$ & 1 里 9 町 $(4,9 \mathrm{~km})$ \\
\hline $\begin{array}{l}\text { 程ヶ谷(保土ヶ谷) } \\
\text { Hodogaya }\end{array}$ & 神奈川県横浜市保土ヶ谷区 & 8 里 9 町 $(32,4 \mathrm{~km})$ & 2 里 9 町 $(8,8 \mathrm{~km})$ \\
\hline 戸塚 Totsuka & 神奈川県横浜市戸塚区 & 10 里 18 町 (41,2 km) & 2 里 $(7,9 \mathrm{~km})$ \\
\hline 藤沢 Fujisawa & 神奈川県藤沢市 & 12 里 18 町 (49,1 km) & 3.5 里 $(13,7 \mathrm{~km})$ \\
\hline 平塚 Hiratsuka & 神奈川県平塚市 & 16 里 $(62,8 \mathrm{~km})$ & 27 町 (2,9 km) \\
\hline 大磯 Ôiso & 神奈川県中郡大磯町 & 16 里 27 町(65,8 km) & 4 里 $(15,7 \mathrm{~km})$ \\
\hline 小田原 Odawara & 神奈川県小田原市 & 20 里 27 町 $(81,5 \mathrm{~km})$ & 4 里 8 町 (16,6 km) \\
\hline 箱根 Hakone & 神奈川県足柄下郡箱根町 & 24 里 35 町 $(98,1 \mathrm{~km})$ & 3 里 28 町 (14,8 km) \\
\hline
\end{tabular}

Sans entrer dans le détail de cette littérature, on peut en retenir une caractéristique profonde de la langue japonaise, et de la culture japonaise en général : une souplesse qui permet de cheminer d'une interprétation à l'autre, d'une situation à l'autre. On a même pu parler d'un « situationnisme» japonais. Cela tient fondamentalement à une structure linguistique et ontologique fort différente de celle qui a permis l'essor du sujet moderne en Occident - cette structure qui a engendré le dualisme, et que Descartes exprime à merveille dans ce passage du Discours de la méthode: " je connus de là que j'étais une substance dont toute l'essence ou la nature n'est que de penser, et qui, pour être, n'a besoin d'aucun lieu, ni ne dépend d'aucune chose matérielle » (Descartes [1637] 2008). Cette transcendance du sujet moderne par rapport à son milieu a été préparée depuis des millénaires par l'existence, dans les langues indo-européennes, d'un pronom « je » qui reste toujours le même, quelle que soit la situation. Au contraire, en japonais, il n'existe pas véritablement de pronoms personnels, et l'expression du sujet varie toujours, suivant la situation (Berque \& Sauzet 2004, Berque 2014). 
C'est dire qu'ontologiquement et linguistiquement, plutôt que le sujet, c'est la situation qui est déterminante. C'est ce qu'on appelle en japonais baai 場合 ou bamen 場面. Corrélativement, le passage, la transition d'une situation à une autre deviennent également déterminants. Tout l'espace-temps en est affecté. La spatialité japonaise accorde ainsi une grande importance aux zones de transition, jouant le rôle d'intermédiaires entre une situation et une autre.

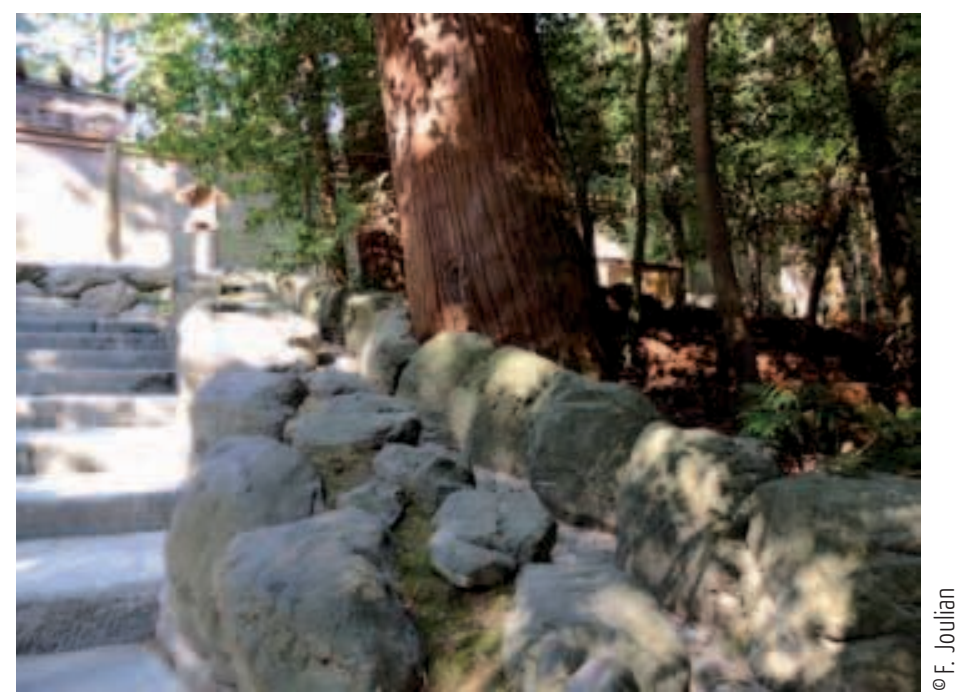

Comparons par exemple la cathédrale Notre-Dame de Paris au sanctuaire d'Ise. Au Moyen Âge, Notre-Dame se dressait immédiatement au contact de la ville, sans zone de transition. On a depuis dégagé une esplanade, qui permet de prendre un certain recul pour mieux admirer la façade. Cela ne change rien au principe : de près ou de loin, la cathédrale est visible et admirable en elle-même. Le sanctuaire d'Ise, au contraire, n'est visible qu'au dernier moment. Pour y arriver, il faut traverser un bois sacré, planté de cèdres millénaires ; et c'est cette transition qui met en valeur le sanctuaire. Je dirai même que marcher sous ces arbres immenses donne une impression de sacralité plus profonde que la vue des bâtiments du sanctuaire. C'est ce que Bashô exprimait dans le poème suivant (Kemmoku \& Chipot 2012) :

$\begin{array}{lll}\text { みそか月なし } & \text { Misoka tsuki nashi } & \text { Fin de mois sans lune } \\ \text { 千とせの杉を } & \text { chitose no sugi wo } & \text { les cèdres millénaires } \\ \text { 抱くあらし } & \text { daku arashi } & \text { étreints par la tempête }\end{array}$

On remarquera que l'auteur, dans ce poème où il exprime pourtant une impression puissante ${ }^{11}$, n'apparaît pas comme sujet. Or ce n'est pas que le sujet soit absent, c'est que son existence est immanente à la scène, au bamen lui-même ; et c'est cela justement qui permet l'élision du sujet grammatical de la phrase. Ce procédé est courant en japonais, alors qu'il est presque inconcevable dans les langues et dans la pensée européennes. En fait, bien que les grammairiens et les philosophes de l'époque Meiji se soient évertués à 
introduire la notion de sujet (dont ils ont rendu les différentes acceptions par shugo 主語, shutai 主体, shukan 主観 etc.), laquelle n'existait pas auparavant dans leur culture (Yanabu 2004, Kobayashi 2010), plutôt que « sujet », c'est « ambiant» qui conviendrait (Berque 1998). Cet « ambiant » (ici l'auteur du poème, Bashô) n'a pas besoin d'être explicité, puisqu'il est implicite. En effet, le japonais ne peut pas se contenter de la structure binaire sujet-prédicat « S est $\mathrm{P}$ ». Par exemple, dans l'énoncé « Marie (S) est triste (P) », ce qui donnerait Mari wa kanashii マリは悲しい, il doit dire Mari wa kanashisô da マリは悲しそ うだ, « Marie semble triste ». Ce n'est

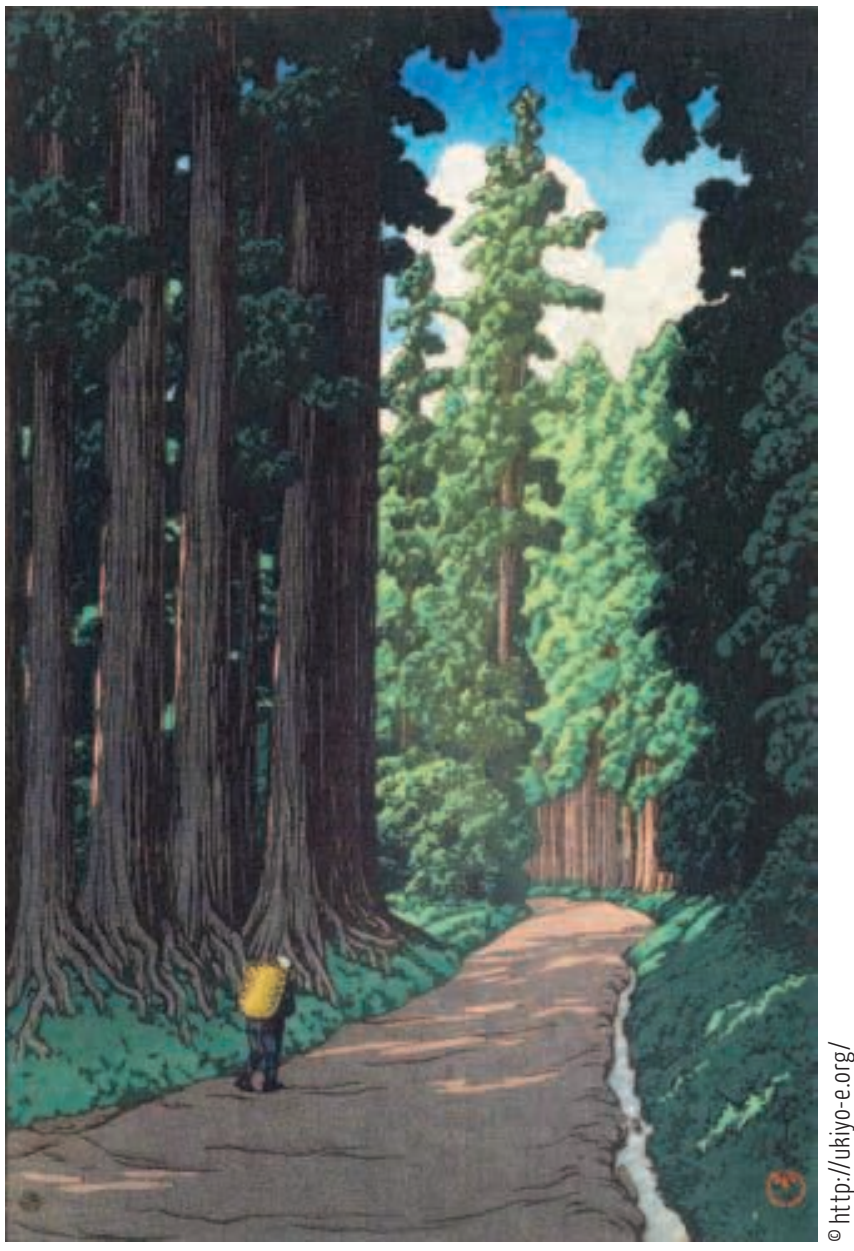

Nikko kaido, Hasui Kawase pas une structure binaire (S-P), d'où l'existence du locuteur est abstraite, mais une structure ternaire, S-I-P, où I est l'ambiant qui interprète la scène, même s'il ne s'explicite pas en un pronom « je » (par exemple comme dans « j'ai l'impression que Marie est triste »).

Lêtre de cet ambiant et l'ambiance du bamen ne peuvent évidemment pas s'opposer comme, chez Descartes, s'opposent la res cogitans (le sujet) et la res extensa (l'objet). L'ambiance est imprégnée de l'être de l'ambiant, et réciproquement. Aussi bien, le passage d'une ambiance à l'autre est-il la grande affaire de l'esthétique japonaise, qui dispose entre autres à cet égard du concept d'utsuroi 移ろい. Dans la langue classique, le verbe utsurou 移ろふ signifie : 《 changer de lieu », « changer de sentiment», « passer (d'un moment à un autre) », « dépérir », «se faner, défraîchir (une couleur) », "se détacher (les pétales des fleurs de cerisier, les feuilles mortes) ». D'où le substantif utsuroi, qui signifie en somme un changement d'ambiance, à la fois dans l'espace et dans le temps.

La notion d'utsuroi a par exemple une grande importance dans les études paysagères (Kobayashi 1993), où le passage du temps peut changer profondément les ambiances. Concernant plus directement la marche, ces passages d'une ambiance à une autre, en allant d'un lieu à un autre, ont été systématiquement mis en valeur, à l'époque d'Édo, dans l'art des jardins-promenades (kaiyû-shiki teien 回遊式庭園), où tout le plaisir de la marche est d'y découvrir une scène après l'autre, par de subtiles transitions où A devient B, l'un devient l'autre. Le plus célèbre de ces jardins est le Koishikawa Kôrakuen 小石川後楽園, dans l'arrondissement de Shinjuku à Tokyo. 
Le Kôrakuen illustre l'inspiration chinoise, et plus particulièrement taoïste, de ces jardins-promenades. Son aménagement final fut réalisé par un lettré chinois, Zhu Shunshui, resté fidèle aux Ming renversés par la dynastie mandchoue des Qing en 1644, et qui s'était réfugié au Japon. Le nom même de Kôrakuen ("Jardin de la réjouissance ultérieure », en chinois Houleyuan) est une citation des Notes de la tour de Yueyang (Yueyanglou-ji岳 陽樓記) de Fan Zhongyan (989-1052), ministre de la dynastie Song ; laquelle signifie dans son intégralité : "S'inquiéter avant que ne s'inquiète l'Empire, se réjouir après que s'est réjoui l'Empire» (Xian Tianxia zhi you er you, hou Tianxia zhi le er le先天下之憂而 憂、後天下之楽而楽 ).

Mais alors qu'en Chine, la spatialité paysagère du jardin de lettré - dont la source est le paradigme érémitique des Six Dynasties - s'oppose à celle de la ville, dominée par une géométrie cosmique, au Japon elle s'étend à toute l'écoumène, dont le terreau shintoïque lui était propice. En effet ce qui, dans les jardins chinois, était un refus de l'ordre géométrique dominant le monde, au Japon était justement cet ordre-monde (kosmos) ; c'est-à-dire une topologie concrète, où les lieux étaient fonction les uns des autres par voisinage, et où la transition de l'un à l'autre s'effectuait au pas humain. C'est cela même que l'on ressent en découvrant à loisir, d'une ambiance à l'autre au fur et à mesure de la marche, les diverses phases du jardin-promenade.

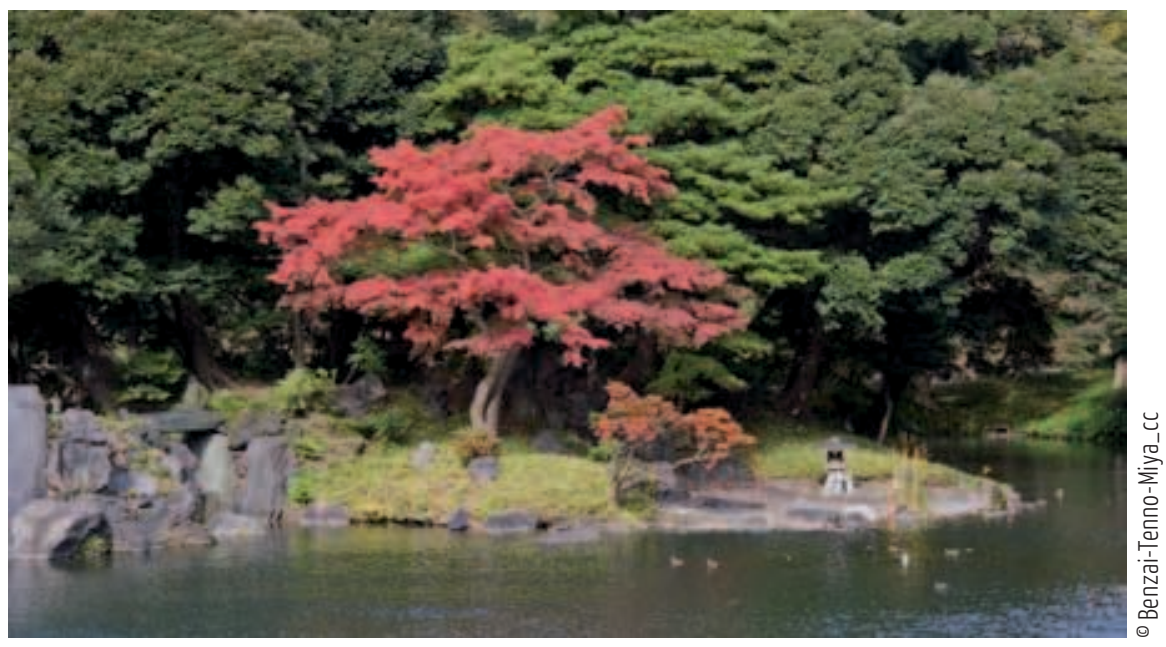

Koishikawa Kôrakuen 


\section{NOTES}

Photo d'ouverture: Bambou de Boudai, parc du Bois de bambous de Kyôto. Cl. Augustin Berque

1. Nom qui veut dire, en grec, «épi feuillu».

2. Les noms japonais sont donnés dans leur ordre normal, patronyme avant le prénom.

3. Kyô 境

4. Jôdo 浄土.

5. Saihô jôdo 西方浄土.

6. Shumisen 須弥山.

7. Zhulin qixian 竹林七賢.

8. Il s'agit de Ruan Ji (210-263), Ji Kang (223-262), Shan Tao (205-285), Liu Ling (? - ?), Ruan Xian (? -?), Xiang Xiu (? -?), et Wang Rong (237-305).

9. Cette phrase maintes fois citée l'est entre autres à la notice Chikurin no shichi ken 竹林の七賢 (Les Sept sages du Bois de bambous ) dans Mô Keien (Meng Qingyuan) E al., 1998.
10. Traduction A. B. Poème reproduit p. 100 (Kemmoku $\&$ Chipot 2012) donnant la traduction suivante: Nuit sans lune de la fin du mois / le vent fort étreint / ce cèdre millénaire.

11. Dans le commentaire qui accompagne ce poème (ibid.), Bashô écrit : «À la tombée de la nuit, j’ai visité le bâtiment extérieur du sanctuaire dédié aux mânes de la famille impériale d'Ise. Les ombres du premier portique, marquant l'entrée du sanctuaire, étaient sombres, et les lanternes sacrées brillaient partout. Me souvenant du "vent de la montagne sacrée qui souffle dans les pins", j'ai été profondément ému ».

\section{RÉFÉRENCES}

Berque, A. 2014 Poétique de la Terre. Histoire naturelle et histoire humaine, essai de mésologie. Paris: Belin.

- 2013 La Marche. In Cycle de conférences de l'automne 2013. Paris : École spéciale d'architecture. http:// ecoumene.blogspot.fr/2013/12/marcher-au-japon-berque.html consulté le 21 novembre 2014.

- 2010 Histoire de l'habitat idéal, de l'Orient vers l'Occident. Paris: Le Félin.

— 1998 « Le japonais ». In Jean-François Mattéi (dir.) Encyclopédie philosophique universelle, IV: le Discours philosophique. Paris: Presses universitaires de France: 240-250.

- [1986] 1997 Le Sauvage et l'artifice. Les Japonais devant la nature. Paris: Gallimard: 200.

Berque, A. \& Sauzet, M. 2004 Le Sens de l'espace au Japon. Vivre, penser, bâtir. Paris: Arguments.

Descartes, R. [1637] 2008 Discours de la méthode. Paris: Flammarion: 38-39.

Grousset, R. 1929 Sur les traces du Bouddha. Paris: Plon.

Jinnai, H. 1995 Tokyo: A Spatial Anthropology. Berkeley: University of California Press.

Kemmoku, M. \& Chipot, D. 2012 Bashô, seigneur ermite. L’intégrale des haïkus. Paris: La Table Ronde: 100.

Kobayashi, T. 2010 "Shutai" no yukue (Destin du "sujet"). Tokyo: Kôdansha: 61.

- 1993 Utsuroi no fûkeiron. Gokan, kotoba, tenki (Changement d'ambiance et paysage. Les sens, les mots, le temps). Tokyo: Kashima Shuppankai.

Lakoff, G. \& Johnson, M. 1999 Philosophy in the flesh. The embodied mind and its challenge to Western thought. New York: Basic Books.

Lévy, A. 1991 Pérégrination vers l'Ouest, trad. de Wu Cheng'En, Xiyouji. Paris: Gallimard (Bibliothèque de la Pléiade, 2 vol.). 
Pigeot, J. 1982 Michiyuki-bun. Poétique de l'itinéraire dans la littérature du Japon ancien. Paris: Maisonneuve et Larose.

Qingyuan, M. \& al. 1998 Chûgoku rekishi bunka jiten (Dictionnaire historique de la culture chinoise). Tokyo: Shinchôsha: 696.

Yanabu, A. 2004 Kindai nihongo no shisô (La pensée du japonais moderne). Tokyo: Hôsei Daigaku Shuppankyoku.

Yoshimura, M. 1976 Kûkan no seitaigaku (Écologie de l'espace). Tokyo: Shôgakkan: 69.

Yoshisada, I. [1969] 2001 Inja no bungaku. Kunô suru bi (La littérature érémitique. Une beauté souffrante). Tokyo: Kôdansha gakujutsu bunko : 116.

\section{POUR CITER CET ARTICLE}

Berque, A. 2014 Marcher au Japon, in S. Boulay \& M.-L. Gélard, Vivre le sable! Corps, matière et sociétés, Techniques \& Culture $61: 254-269$

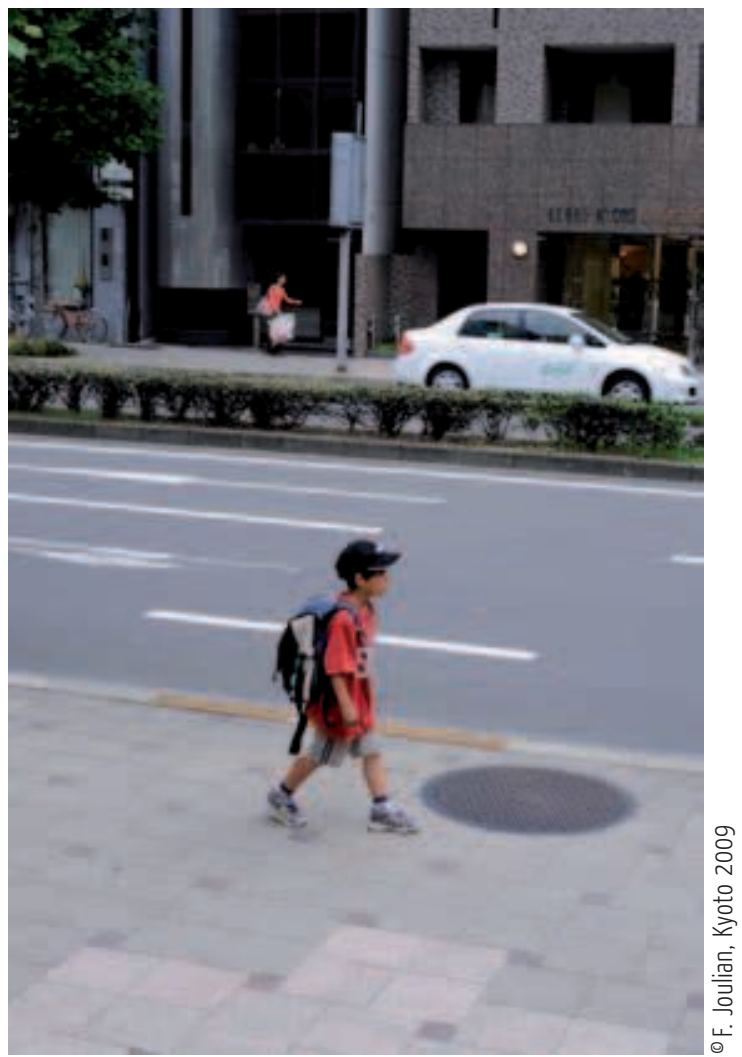

